\title{
Applicability of electrospun polypropylene carbonate polymers as a drug carrier for sirolimus
}

\author{
HOURONG SUN, XINGHUA GU, KAI LIU, CHANGCUN FANG and MENGMENG TANG \\ Department of Cardiovascular Surgery, Qilu Hospital, Shandong University, Jinan, Shandong 250012, P.R. China
}

Received April 6, 2016; Accepted March 8, 2017

DOI: $10.3892 / \mathrm{mmr} .2017 .6540$

\begin{abstract}
Polypropylene carbonate (PPC), a biodegradable aliphatic polyester, exhibits one particular advantage over other polyesters, which is that following degradation in vivo, it primarily produces $\mathrm{H}_{2} \mathrm{O}$ and $\mathrm{CO}_{2}$, causing minimal side effects. Although PPC exhibits limited mechanical strength, and is therefore not able to serve as a scaffold to support tissue regeneration, it may be suitable for drug delivery; however, this requires further investigation. In the present study, electrospinning was applied to generate PPC polymers containing sirolimus, a cell growth-inhibiting drug which is used to treat restenosis. The properties of PPC-sirolimus polymers were examined using scanning electron microscopy, differential scanning calorimetry and in vitro degradation assays. Drug loading and entrapment efficiency were determined, and in vitro sirolimus-release from the polymer was assessed. Furthermore, the effect of PPC-sirolimus polymers on cell growth was measured using an MTT assay in vitro. The results of the present study demonstrated that electrospun PPC polymers formed a uniform three-dimensional, grid-intertwined, net-like structure; the surface of the polymers was smooth and the diameter was $\sim 3 \mu \mathrm{m}$. Differential scanning calorimetry analysis demonstrated that sirolimus existed in an amorphous state in the polymer. Following soaking in PBS for 4 weeks, the polymer swelled and the net-like structure broke down and fragmented. Sirolimus loading and entrapment efficiency were $10.3 \pm 3.2$ and $95.1 \pm 10.6 \%$, respectively. Sirolimus-release from PPC-sirolimus polymers continued for 28 days in PBS. PPC-sirolimus markedly inhibited the growth of rat aortic adventitial fibroblast cells, an effect which was not observed with PPC alone. The results of the present study suggest that PPC polymers are a promising alternative drug carrier for sirolimus.
\end{abstract}

Correspondence to: Dr Xinghua Gu, Department of Cardiovascular Surgery, Qilu Hospital, Shandong University, 107 Wenhua West Road, Jinan, Shandong 250012, P.R. China

E-mail: guxinghuagxh@sina.com

Key words: polypropylene carbonate, sirolimus, electrospinning, drug carrier, biodegradable, cell growth

\section{Introduction}

Polymers may be naturally-occurring, including collagen and polysaccharides, or synthesized, including polylactide (PLA), polyethylene and poly lactic-co-glycolic acid (PLGA) (1-7). Polymers have been extensively studied and used for biomedical and pharmaceutical applications $(1,8)$. As polymer synthesis may be conducted under controlled conditions, and the properties of polymers, including hydrophilicity, degradability and biocompatibility, may be tailored for specific applications, numerous studies have focused on the development of novel synthetic polymers for tissue-engineering (polymers as scaffolds for tissue regeneration) and controlled drug release (polymers as drug carriers) $(3,4,9)$. Additionally, synthetic polymers are frequently less expensive as they may be synthesized in large quantities and exhibit long shelf lives (4).

Aliphatic polyesters, including PLA, polyglycolide (PGA) and PLGA, are among the most commonly used synthetic polymers in medicine, due to their excellent biocompatibility and biodegradability (10). PLA is used for manufacturing sutures and bone screws; PLA, PGA and particularly PLGA are being investigated for drug delivery applications $(7,11,12)$. Polypropylene carbonate (PPC), a relatively new aliphatic polyester, is synthesized via the copolymerization of $\mathrm{CO}_{2}$ and propylene oxide $(13,14)$. It is known that PPC possesses limited mechanical strength, and is therefore not suited to serve as a scaffold to support tissue regeneration (10), although it may be suitable for drug delivery; however, this requires further investigation.

Electrospinning has been used for the fabrication of polymer fibers (15-17). Electrospinning uses an electric field, wherein a polymer solution is charged and driven by the electric potential. When the charged jet of polymer solution travels across an air gap, the solvent evaporates while the polymer fibers, with diameters ranging between nanometers and micrometres, are generated. In the present study, the applicability of the PPC polymer as a drug carrier for sirolimus, also known as rapamycin, was investigated. Sirolimus is a cell growth-inhibiting agent used to treat vascular restenosis. Electrospinning was employed to synthesize PPC polymer containing sirolimus. The properties of the drug-containing PPC polymer were investigated, drug loading and entrapment efficiency were determined, and in vitro sirolimus release was assessed. In addition, the effect of the PPC-sirolimus polymer on cell growth was studied in vitro. 


\section{Materials and methods}

Materials. PPC (MW 100,000; purity $>99 \%$ ) was purchased from Inner Mongolia Mengxi High Tech Group Co., Ltd. (Ordos, China). Sirolimus (rapamycin) was obtained from Fujian Kerui Pharmaceutical Co., Ltd. (Fuzhou, China). All chemicals were from Jiete'ao Biotechnology Co., Ltd. (Beijing, China). MTT was purchased from Sigma-Aldrich (Merck KGaA, Darmstadt, Germany). Eagle's minimum essential medium (EMEM), fetal bovine serum (FBS), penicillin and streptomycin were obtained from Gibco (Thermo Fisher Scientific, Inc., Waltham, MA, USA).

Preparation of PPC polymer loaded with sirolimus. Various ratios of PPC and sirolimus were used to produce PPC-sirolimus polymers and cell growth experiments, performed by MTT assay as previously described (18), demonstrated that the polymer made with $900 \mathrm{mg}$ PPC and $100 \mathrm{mg}$ sirolimus resulted in the greatest cell inhibitory effect; therefore, this ratio was used in the present study. The polymer solution was as follows: $1,000 \mathrm{mg}$ PPC alone (PPC polymer) or $900 \mathrm{mg}$ PPC plus $100 \mathrm{mg}$ sirolimus (PPC-sirolimus polymer) was dissolved in $10 \mathrm{ml}$ acetonitrile following stirring for $6 \mathrm{~h}$. Electrospinning was performed using the electrospinning apparatus (Model ET2535; Beijing Ucalery Technology and Development Co., Ltd., Beijing, China). The polymer solution was transferred into a syringe attached with a blunt tipped needle. The anode of the power supply was clamped to the needle tip and the cathode was connected to the grounded collector plate (Fig. 1). Electrospinning was performed according to the established protocol (Institute of Polymer Science, Tsinghua University, Beijing, China) (19).

Scanning electron microscopy (SEM). The polymers were coated with silver using the vacuum evaporator (HUS 5GB; Hitachi Ltd., Tokyo, Japan) following an established protocol at the Imaging Center, Shandong University (Jinan, China) (20). The silver coated polymers were analyzed using a scanning electron microscope (H-8010; Hitachi, Ltd., Japan) operated at $15 \mathrm{kV}$, and images were captured.

Differential scanning calorimetry (DSC). The thermal behavior of the PPC-sirolimus polymers was assessed by DSC analysis using the DSC822e differential scanning calorimeter (Mettler Toledo, Greifensee, Switzerland). A total of $4 \mathrm{mg}$ sample (pure sirolimus or PPC-sirolimus polymer) was weighed in a $40 \mu 1$ aluminum pan and placed in the sample chamber of the calorimeter, and an empty pan was used as the reference. A flow of nitrogen gas was maintained over the sample to eliminate air oxidation of the sample at high temperatures, and to create a reproducible and dry atmosphere. DSC analysis was performed using the STARe software version 8.10 (Mettler Toledo) with the temperature range $20-300^{\circ} \mathrm{C}$ and the temperature increase being set at a rate of $10^{\circ} \mathrm{C} / \mathrm{min}$. The endothermic or exothermic peak was automatically recorded.

In vitro PPC-sirolimus polymer degradation assessment. A PPC-sirolimus polymer of $1 \times 1 \mathrm{~cm}$ was placed in a $5-\mathrm{ml}$ test tube and soaked in PBS (pH 7.2). The tube was gently shaken
$(60 \mathrm{rpm})$ at $37^{\circ} \mathrm{C}$ for 4 weeks. Subsequently, the structure of the polymer was examined using SEM.

Determination of drug loading and entrapment efficiency. The efficiency of drug loading and entrapment was determined as follows: $1,000 \mathrm{mg}$ PPC-sirolimus polymer was dissolved in dichloromethane by stirring for $2 \mathrm{~h}$ followed by addition of $0.1 \mathrm{M}$ hydrochloric acid. Following mixing by vortex, lower dichloromethane and upper hydrochloric acid layers were formed. The hydrochloric acid layer was collected, centrifuged at $1,000 \mathrm{x} \mathrm{g}$ for $5 \mathrm{~min}$ at room temperature and the supernatant was collected. The precipitate was mixed with $0.1 \mathrm{M}$ hydrochloric acid containing 0.1\% EDTA. When the precipitate was completely dissolved, the solution was added to the previously collected hydrochloric acid supernatant. The sirolimus in the combined solution was measured by high pressure liquid chromatography as described previously (21). The sirolimus loading efficiency (\%) was calculated using the following formula: (Total sirolimus amount in the combined solution/polymer weight) x 100. Drug entrapment efficiency was calculated using the following formula: [Total sirolimus amount in the combined solution/total sirolimus amount used for polymer preparation $(100 \mathrm{mg})] \mathrm{x} 100$. A total of three PPC-sirolimus polymer preparations were used to determine the drug loading and entrapment efficiency.

In vitro measurement of sirolimus release from polymers. A total of $5 \mathrm{mg}$ PPC-sirolimus polymer was placed in 14 separate 5 - $\mathrm{ml}$ test tubes. The polymer was immersed in $4 \mathrm{ml} \mathrm{PBS/tube}$ and gently shaken $(60 \mathrm{rpm})$ at $37^{\circ} \mathrm{C}$. At day $2,4,6,8,10,12$, $14,16,18,20,22,24,26$ and 28 following shaking, the polymer was removed and the sirolimus contained in the polymer was isolated and measured as described in the determination of drug loading and entrapment efficiency section. At each time point, the amount of released sirolimus was calculated using the following formula: Total sirolimus in the original $5 \mathrm{mg}$ polymer-total sirolimus in the soaked polymer. The release percentage was calculated using the following formula: (Amount of released sirolimus/total sirolimus in the original $5 \mathrm{mg}$ polymer) x 100.

Inhibition of cell growth by PPC-sirolimus polymers. An MTT assay was performed to examine the anti-cell proliferative effect of the PPC-sirolimus polymer. Rat aortic adventitial fibroblast cells (RAAFCs) were isolated as described previously $(22,23)$. A total of 4 Wistar rats $(2$ males and 2 females, $\sim 7$ weeks old, weighing 180-220 g) were used for RAAFC isolation. The animals were purchased from Shandong Experimental Animal Center (Jinan, China), housed in the Shandong University (Jinan, China) Animal Room with temperature at $20 \pm 2^{\circ} \mathrm{C}$, and fed with standard chow. The use of animals was approved by the Research Ethics Committee of Shandong University in compliance with the Guidelines for the Care and Use of Laboratory Animals issued by the Ministry of Science and Technology, China. Cells were cultured in EMEM with $10 \% \mathrm{FBS}, 50 \mathrm{IU} / \mathrm{ml}$ penicillin and $100 \mu \mathrm{g} / \mathrm{ml}$ streptomycin at $37^{\circ} \mathrm{C}$ in a humidified atmosphere containing $5 \% \mathrm{CO}_{2}$. For the MTT assay, $5 \times 10^{3}$ cells suspended in $100 \mu \mathrm{l}$ culture medium were seeded into each well of a 96-well plate, into which had previously been added $2 \mathrm{mg}$ of PPC or 


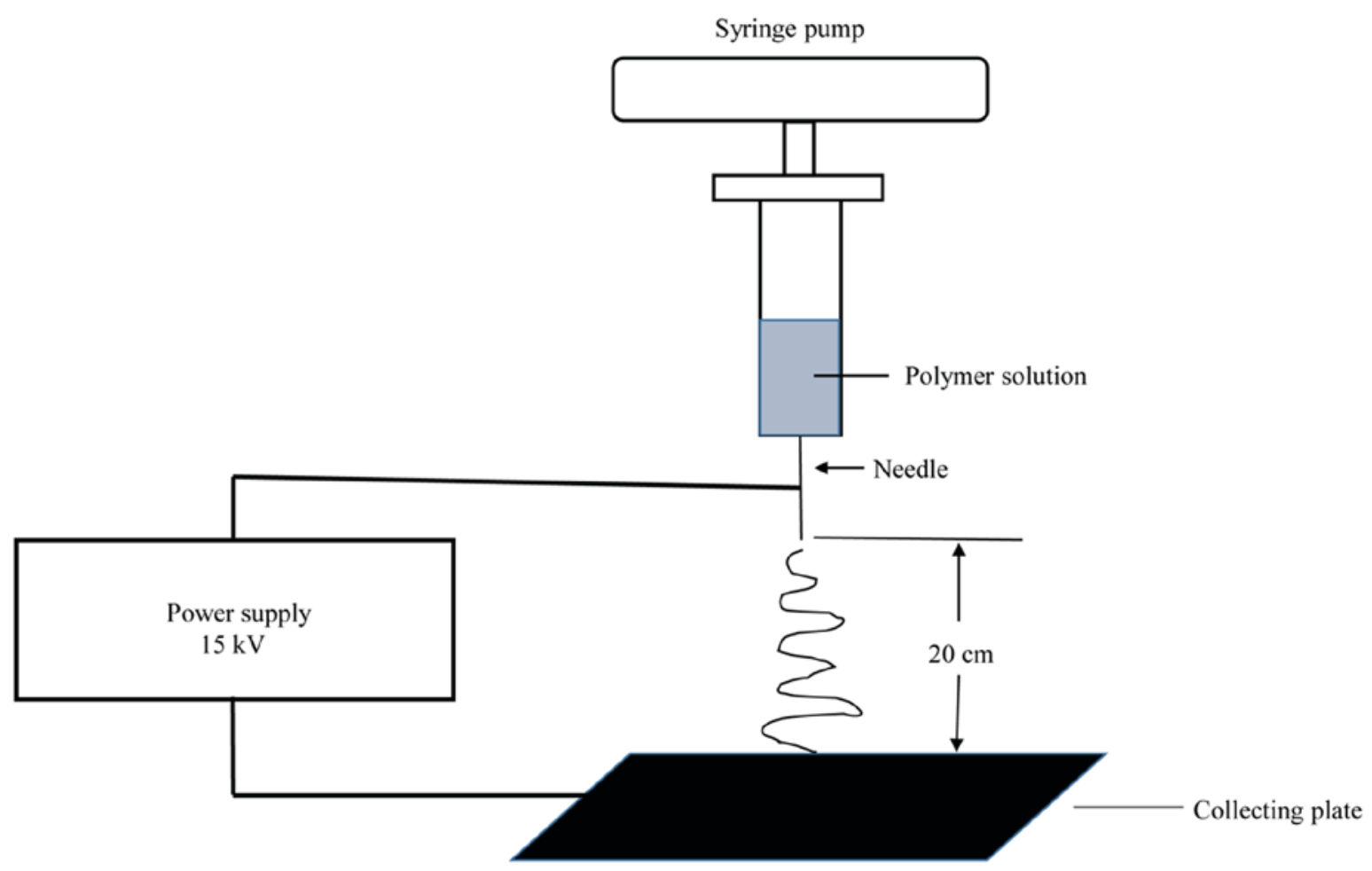

Figure 1. Schematic diagram of electrospinning apparatus. Polymer solution was placed in the syringe. Electrospinning was performed with the voltage being set at $15 \mathrm{kV}$ and the distance of the needle tip to the collector being set at $20 \mathrm{~cm}$.

PPC-sirolimus polymer (each sample in triplicate). Cells seeded in wells with no polymer were taken as the control. Following culturing for 1 and 3 days, the MTT assay was performed as follows: $10 \mu 1 \mathrm{MTT}$ solution was added into each well and incubated at $37^{\circ} \mathrm{C}$ for $4 \mathrm{~h}$, followed by the addition of $50 \mu \mathrm{l}$ dimethyl sufoxide/well. Following gentle shaking for $10 \mathrm{~min}$, absorbance at a wavelength of $570 \mathrm{~nm}$ was measured using a 96-well plate reader (Model 680, Bio-Rad Laboratories, Inc., Hercules, CA, USA). The relative cell viability (\%) of each sample was calculated using the following formula: (Average sample absorbance/average control absorbance) x 100 .

Statistical analysis. Cell growth data are expressed as the mean \pm standard error of the mean. The SPSS 17.0 software (SPSS, Inc., Chicago, IL, USA) was used for statistical analysis. Statistical significance was determined using the Student's t-test. $\mathrm{P}<0.05$ was considered to indicate a statistically significant difference.

\section{Results}

Electrospinning and polymer characterization. Electrospinning conditions were optimized and used as follows: The voltage was set at $15 \mathrm{kV}$; the distance from the needle tip to the collector was $20 \mathrm{~cm}$, and the flow rate of the spinning solution was controlled at $0.2 \mathrm{ml} / \mathrm{h}$ by a syringe pump (TJ-3A/W0109-1B; Longer Precision Pump Co., Ltd., Baoding, China) (Fig. 1). SEM analysis demonstrated that the polymer exhibited a regular three-dimensional, grid-intertwined, net-like structure with a smooth surface, and the diameter was $\sim 3 \mu \mathrm{m}$ (Fig. 2). DSC analysis demonstrated that the crystalline pure sirolimus produced an endothermic peak at $\sim 230^{\circ} \mathrm{C}$, the melting point of

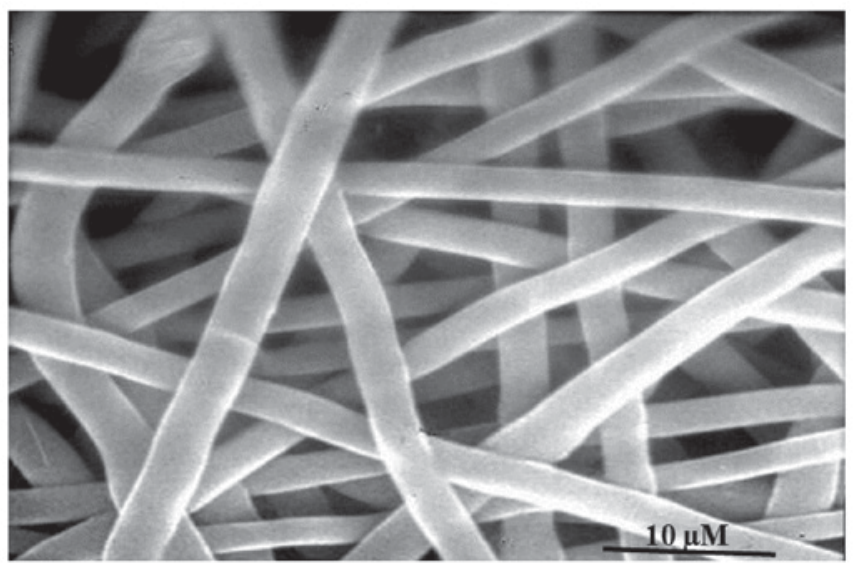

Figure 2. Scanning electron microscopy analysis. The polypropylene carbonate-sirolimus polymer exhibited a uniform three-dimensional, grid-intertwined, net-like structure with a smooth surface, and the diameter was $\sim 3 \mu \mathrm{m}$

sirolimus (Fig. 3A). By contrast, the PPC-sirolimus polymer exhibited a glass transition temperature of $\sim 40^{\circ} \mathrm{C}$ for PPC (Fig. 3B). The in vitro degradation experiment demonstrated that, following soaking in PBS for 4 weeks, the polymer swelled and the regular three-dimensional grid-intertwined structure broke down and fragmented (Fig. 4).

Sirolimus loading, entrapment efficiency and release from polymer. Sirolimus loading and entrapment efficiency were $10.3 \pm 3.2$ and $95.1 \pm 10.6 \%$, respectively. The release of sirolimus was measured for 28 days. As presented in Fig. 5, sirolimus was gradually released from the PPC-sirolimus polymer in a 

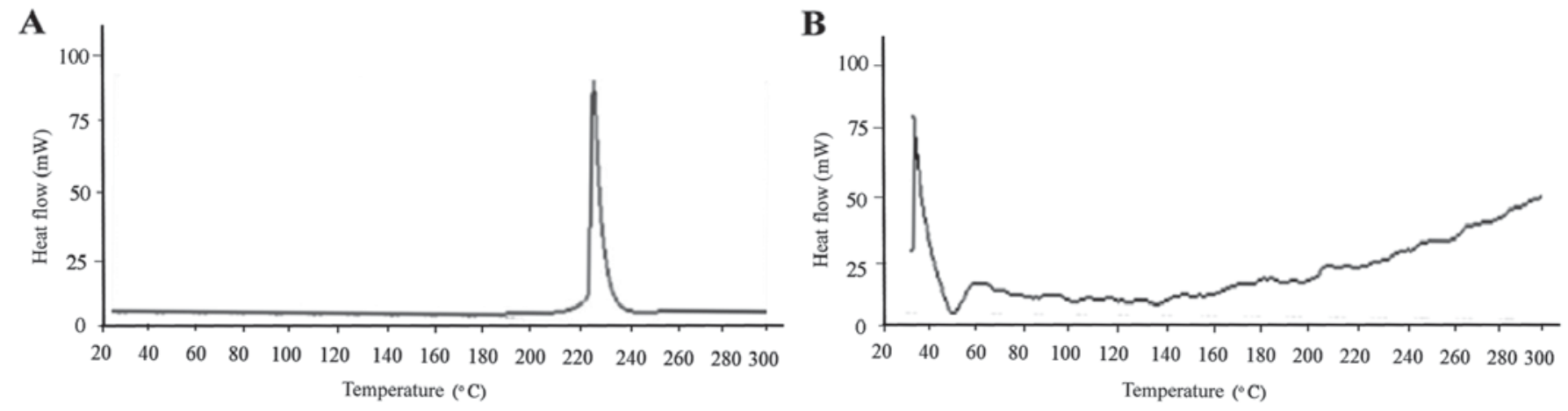

Figure 3. Thermograms of differential scanning calorimetry. (A) The crystalline pure sirolimus produced an endothermic peak at $\sim 230^{\circ} \mathrm{C}$, the melting point of Sirolimus. (B) The polypropylene carbonate-sirolimus polymer exhibited a glass transition temperature of $\sim 40^{\circ} \mathrm{C}$.

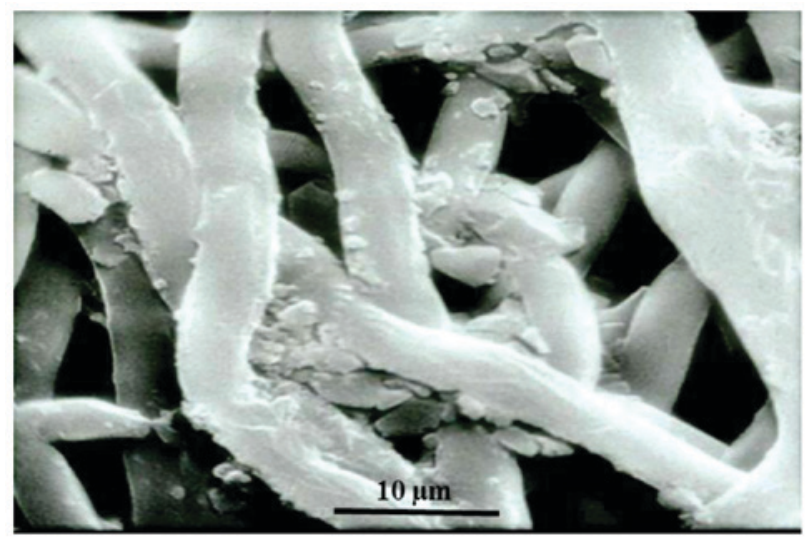

Figure 4. In vitro degradation analysis. Following soaking in PBS for 28 days, the polypropylene carbonate-sirolimus polymer swelled, became larger, and the grid-intertwined structure broke down and fragmented.

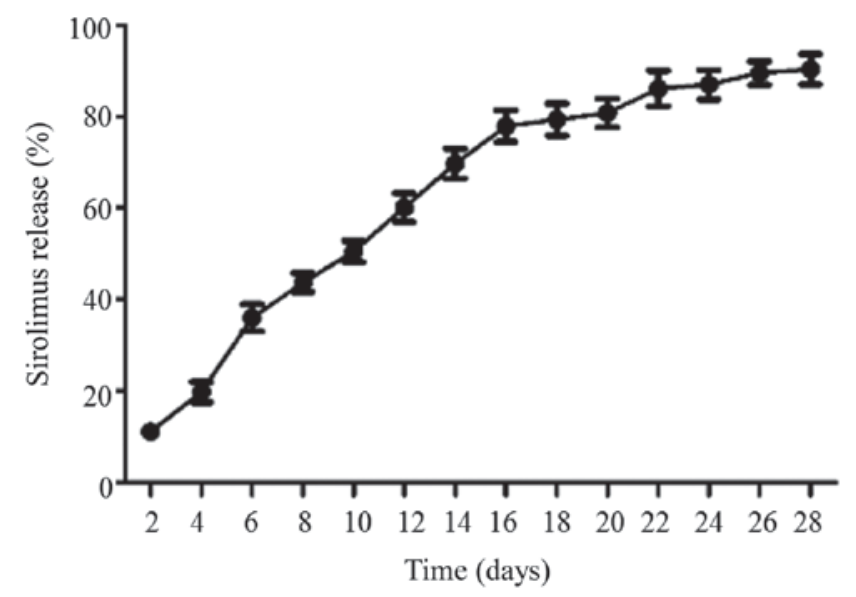

Figure 5. Sirolimus release assay. The drug release from the polypropylene carbonate-sirolimus polymer occurred in a linear-like fashion, particularly for the first 3 weeks, and continued for 28 days.

linear-like fashion, particularly for the first 3 weeks. A total of $\sim 90 \%$ of the sirolimus entrapped in the polymer was released after 28 days.

Inhibition of RAAFC growth by the PPC-sirolimus polymer. An MTT assay was performed in order to evaluate the effect of the PPC-sirolimus polymer on RAAFC growth. The

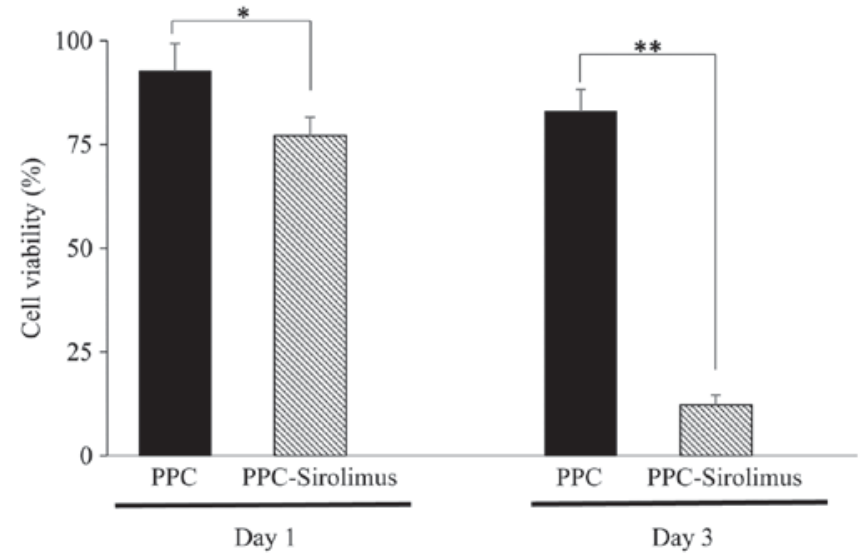

Figure 6. PPC-sirolimus polymer inhibits RAAFC growth. The PPC polymer did not affect the growth of RAAFCs significantly. By contrast, coculture with PPC-sirolimus polymer for 1 day or 3 days led to a decrease in cell viability. ${ }^{*} \mathrm{P}<0.05,{ }^{* *} \mathrm{P}<0.01$ vs. $\mathrm{PPC}$ alone. $\mathrm{PPC}$, polypropylene carbonate; RAAFCs, rat aortic adventitial fibroblast cells.

viability of control cells (without the addition of any polymer) was arbitrarily set at $100 \%$. As presented in Fig. 6, following culture for 1 day, the viability of cells treated with PPC and PPC-sirolimus was $92.7 \pm 6.7$ and $77.2 \pm 4.4 \%$, respectively. At day 3 , the viability of cells treated with PPC and PPC-sirolimus was $82.9 \pm 5.4$ and $12.2 \pm 2.4 \%$, respectively. Statistical analysis demonstrated that the PPC-sirolimus polymer significantly inhibited the growth of RAAFCs at the two time points, while the PPC polymer did not (Fig. 6).

\section{Discussion}

Subsequent to the first investigations into polymer-controlled drug release in cancer therapy $(8,24,25)$, the field has rapidly expanded and applied to the treatment of other diseases $(18,26,27)$. Aliphatic polyesters, particularly PLGA, have been extensively explored for local drug delivery. However, one disadvantage of traditional aliphatic polyesters is that they generate acidic products following degradation in vivo, which may cause aseptic inflammation and tissue necrosis $(10,28-30)$. By contrast, PCC, a novel aliphatic polyester, is distinct in that it primarily produces $\mathrm{H}_{2} \mathrm{O}$ and $\mathrm{CO}_{2}$ following degradation, eliminating the side effects caused by PLA, PGA and PLGA (14). Additionally, PPC exhibits an 
increased bioadhesive capacity compared with PLA, PGA and PLGA (21), enabling PPC-drug conjugates to be retained in the target area and exert prolonged therapeutic effects.

Using electrospinning technology, PPC fibers containing sirolimus were generated in the present study. As examined by SEM, the PPC-sirolimus polymer formed a uniform three-dimensional and grid-intertwined net-like structure. The structure formed in electrospun fibers is hypothesized to exhibit a large surface area which is ideal for drug release (31). DSC analysis demonstrated that the endothermic peak for sirolimus was not present in PPC-sirolimus polymer, indicating that sirolimus may exist in the polymer in an amorphous state. Notably, DSC sensitivity may be affected by the low concentration of the drug in the polymer fibers. It is thought that the amorphous state enhances the release of the drug from the polymers (32). In a previous study, PLGA was used to load sirolimus, and the entrapment and drug loading efficiencies were reported to be $\sim 80$ and $\sim 5 \%$, respectively (21), slightly lower compared with what was observed with PPC in the present study.

It is desirable that entrapped therapeutic agents in a polymer are able to be released for a period of time, thus maintaining a sustained action and decreasing the number of administration procedures required. The results of the present study demonstrate that sirolimus encapsulated in a PPC polymer was released in a linear-like manner, particularly for the first 3 weeks. At week 4, 90\% of the sirolimus had been released. Zou et al (21) demonstrated the application of PLGA with carbopol, a compound which enhances PLGA bioadhesion, for local sirolimus delivery; it was observed in an in vitro experiment that sirolimus was able to be released for 4 weeks. Kang et al (33) observed that the release of doxorubicin from polyorganophosphazene hydrogels lasted 20-30 days, which is consistent with the results of the present study. In vitro experiments in the present study demonstrated that PPC did not affect the growth of RAAFCs. However, the PPC-sirolimus polymer significantly reduced cell viability, warranting further in vivo investigation of the effects of PPC-sirolimus.

In conclusion, compared with PLGA, the most widely studied aliphatic polyester for drug delivery, PPC exhibits a similar drug release curve and slightly increased loading and entrapment efficiency with respect to sirolimus encapsulation. As PPC exhibits increased bioadhesion and primarily produces $\mathrm{H}_{2} \mathrm{O}$ and $\mathrm{CO}_{2}$ following degradation in vivo, causing minimal side effects, PPC may be a promising alternative polymer to PLGA for controlled drug delivery.

\section{Acknowledgements}

The present study was supported by the Independent Innovation Foundation of Shandong University (grant no. 2012TS171) and the Research Award Fund for Outstanding Young Scientists of Shandong Province (grant no. 2006BS03014).

\section{References}

1. Lendlein A: Polymers in biomedicine. Macromol Biosci 10 993-997, 2010

2. Chattopadhyay S and Raines RT: Review collagen-based biomaterials for wound healing. Biopolymers 101: 821-833, 2014.

3. Williams CK: Synthesis of functionalized biodegradable polyesters. Chem Soc Rev 36: 1573-1580, 2007.
4. Gunatillake P, Mayadunne R and Adhikari R: Recent developments in biodegradable synthetic polymers. Biotechnol Annu Rev 12: 301-347, 2006.

5. Fambri L, Pegoretti A, Fenner R, Incardona SD and Migliaresi C: Biodegradable fibres of poly(L-lactic acid) produced by melt spinning. Polymer 38: 79-85, 1997.

6. Peyton SR, Raub CB, Keschrumrus VP and Putnam AJ: The use of poly(ethylene glycol) hydrogels to investigate the impact of ECM chemistry and mechanics on smooth muscle cells. Biomaterials 27: 4881-4893, 2006.

7. Kapoor DN, Bhatia A, Kaur R, Sharma R, Kaur G and Dhawan S: PLGA: A unique polymer for drug delivery. Ther Deliv 6: 41-58, 2015.

8. Langer RS and Peppas NA: Present and future applications of biomaterials in controlled drug delivery systems. Biomaterials 2 : 201-214, 1981.

9. Middleton JC and Tipton AJ: Synthetic biodegradable polymers as orthopedic devices. Biomaterials 21: 2335-2346, 2000.

10. Seyednejad H, Ghassemi AH, van Nostrum CF, Vermonden T and Hennink WE: Functional aliphatic polyesters for biomedical and pharmaceutical applications. J Control Release 152: 168-176, 2011.

11. Cameron DJ and Shaver MP: Aliphatic polyester polymer stars: Synthesis, properties and applications in biomedicine and nanotechnology. Chem Soc Rev 40: 1761-1776, 2011.

12. Jain R, Shah NH, Malick AW and Rhodes CT: Controlled drug delivery by biodegradable poly(ester) devices: Different preparative approaches. Drug Dev Ind Pharm 24: 703-727, 1998.

13. Li XH, Meng YZ, Chen GQ and Li RKY: Thermal properties and rheological behavior of biodegradable aliphatic polycarbonate derived from carbon dioxide and propylene oxide. J Appl Polym Sci 94: 711-716, 2004.

14. Zhong X and Dehghani F: Solvent free synthesis of organometallic catalysts for the copolymerisation of carbon dioxide and propylene oxide. Appl Catal B 98: 101-111, 2010.

15. Li WJ, Laurencin CT, Caterson EJ, Tuan RS and Ko FK: Electrospun nanofibrous structure: A novel scaffold for tissue engineering. J Biomed Mater Res 60: 613-621, 2002.

16. Ji Y, Ghosh K, Shu XZ, Li B, Sokolov JC, Prestwich GD, Clark RA and Rafailovich MH: Electrospun three dimensional hyaluronic acid nanofibrous scaffolds. Biomaterials 27: 3782-3792, 2006.

17. Tipduangta P, Belton P, Fábián L, Wang LY, Tang H, Eddleston $M$ and Qi S: Electrospun polymer blend nanofibers for tunable drug delivery: The role of transformative phase separation on controlling the release rate. Mol Pharm 13: 25-39, 2016.

18. Zheng L, Chen J, Ma Z, Liu W, Yang F, Yang Z, Wang K, Wang X, $\mathrm{He}$ D, Li L and Zeng J: Capsaicin enhances anti-proliferation efficacy of pirarubicin via activating TRPV1 and inhibiting PCNA nuclear translocation in 5637 cells. Mol Med Rep 13: 881-887, 2016.

19. Zeng J, Chen X, Xu X, Liang Q, Bian X, Yang L and Jing X: Ultrafine fibers electrospun from biodegradable polymers. J Appl Polym Sci 89: 1085-1092, 2003.

20. Ni S, Xia T, Li X, Zhu X, Qi H, Huang S and Wang J: Sustained delivery of chondroitinase $\mathrm{ABC}$ by poly(propylene carbonate)-chitosan micron fibers promotes axon regeneration and functional recovery after spinal cord hemisection. Brain Res 1624: 469-478, 2015.

21. Zou W, Cao G, Xi Y and Zhang N: New approach for local delivery of rapamycin by bioadhesive PLGA-carbopol nanoparticles. Drug Deliv 16: 15-23, 2009.

22. Gu M and Brecher P: Nitric oxide-induced increase in p21(Sdi1/Cip1/Waf1) expression during the cell cycle in aortic adventitial fibroblasts. Arterioscler Thromb Vasc Biol 20: 27-34, 2000.

23. Tsuruda T, Kato J, Cao YN, Hatakeyama K, Masuyama H, Imamura T, Kitamura K, Asada Y and Eto T: Adrenomedullin induces matrix metalloproteinase-2 activity in rat aortic adventitial fibroblasts. Biochem Biophys Res Commun 325: 80-84, 2004.

24. Duncan R: Polymer conjugates as anticancer nanomedicines. Nat Rev Cancer 6: 688-701, 2006.

25. Davis ME, Chen Z and Shin DM: Nanoparticle therapeutics: An emerging treatment modality for cancer. Nat Rev Drug Discov 7: 771-782, 2008.

26. Liechty WB, Kryscio DR, Slaughter BV and Peppas NA: Polymers for drug delivery systems. Annu Rev Chem Biomol Eng 1: 149-173, 2010.

27. Merkle HP: Drug delivery's quest for polymers: Where are the frontiers? Eur J Pharm Biopharm 97: 293-303, 2015. 
28. Fu K, Pack DW, Klibanov AM and Langer R: Visual evidence of acidic environment within degrading poly(lactic-co-glycolic acid) (PLGA) microspheres. Pharm Res 17: 100-106, 2000.

29. Danmark S, Finne-Wistrand A, Schander K, Hakkarainen M, Arvidson K, Mustafa $\mathrm{K}$ and Albertsson AC: In vitro and in vivo degradation profile of aliphatic polyesters subjected to electron beam sterilization. Acta Biomater 7: 2035-2046, 2011.

30. Ding AG and Schwendeman S: Acidic microclimate $\mathrm{pH}$ distribution in PLGA microspheres monitored by confocal laser scanning microscopy. Pharm Res 25: 2041-2052, 2008.
31. Zamani M, Prabhakaran MP and Ramakrishna S: Advances in drug delivery via electrospun and electrosprayed nanomaterials. Int J Nanomedicine 8: 2997-3017, 2013.

32. Zahedi P and Lee PI: Solid molecular dispersions of poorly water-soluble drugs in poly(2-hydroxyethyl methacrylate) hydrogels. Eur J Pharm Biopharm 65: 320-328, 2007.

33. Kang GD, Cheon SH and Song SC: Controlled release of doxorubicin from thermosensitive poly(organophosphazene) hydrogels. Int J Pharm 319: 29-36, 2006. 